\title{
Asymptotic behaviors for eigenvalues and eigenfunctions associated to Stokes operator in the presence of small boundary perturbations
}

\author{
Christian Daveau * $\quad$ Abdessatar Khelifi ${ }^{\dagger}$
}

December 22, 2016

\begin{abstract}
We consider the Stokes eigenvalue problem in a bounded domain of $\mathbb{R}^{3}$ with Dirichlet boundary conditions. The aim of this paper is to advance the development of high-order terms in the asymptotic expansions of the boundary perturbations of eigenvalues, eigenfunctions and eigenpressures for the Stokes operator caused by small perturbations of the boundary. Our derivation is rigorous and proved by layer potential techniques.
\end{abstract}

Key words. Stokes eigenvalue problem, small perturbation, asymptotic expansion

2010 AMS subject classifications. 47A75, 35P05, 35Q30.

\section{Introduction}

The field of eigenvalue problems under shape perturbation has been an active research area for several decades. Several related problems belong to Stokes systems, which are further subdivided by assumptions on the underlying media and on the Dirichlet boundary conditions. The main objective of this paper is to present a schematic way to derive high-order asymptotic expansions for both eigenvalues and eigenfunctions for the Stokes operator caused by small perturbations of the boundary. The properties of eigenvalue problems under shape deformation have been a subject of comprehensive studies [1, 2, 15, 13] and the area continues to carry great importance [8, 10, 12, 14, 9]. A substantial portion of these investigations discusses the properties of smoothness and analyticity of eigenvalues and eigenfunctions with respect to perturbations.

*Department of Mathematics, CNRS (UMR 8088), University of Cergy-Pontoise, 2 avenue Adolphe Chauvin, 95302 Cergy-Pontoise Cedex, France. (Email: christian.daveau@u-cergy.fr)

†Département de Mathématiques, Université des Sciences de Carthage, Bizerte, Tunisie. (Email: abdessatar.khelifi@fsb.rnu.tn) 
Let $\Omega \subset \mathbb{R}^{3}$ be a bounded open domain with boundary of class $C^{2}$. We consider the following eigenvalue problem for the Stokes system with homogeneous boundary conditions:

$$
\begin{cases}-\Delta v+\nabla \cdot p=\lambda v & \text { in } \Omega \\ \nabla \cdot v=0 & \text { in } \Omega \\ v=0 & \text { in } \partial \Omega .\end{cases}
$$

Here $v=\left(v_{1}, v_{2}, v_{3}\right)$ denotes the velocity field, while the scalar function $p$ is the pressure.

It is well known that this eigenvalue problem admits a sequence of a no decreasing positive eigenvalues $0<\lambda_{1} \leq \lambda_{2} \leq \cdots \leq \lambda_{n} \leq \cdots$ tending to infinity as $n \rightarrow+\infty$.

The eigenfunctions $\left\{v_{n}\right\}_{n \geq 1} \subset\left(H_{0}^{1}(\Omega)\right)^{2}$ and the eigenpressures $\left\{p_{n}\right\}_{n \geq 1} \subset L^{2}(\Omega)$ may be taken so that $\left\{v_{n}\right\}_{n \geq 1}$ constitutes an orthonormal basis of

$$
H(\Omega):=\left\{v \in\left(L^{2}(\Omega)\right)^{3}: \quad \nabla \cdot v=0 \text { in } \Omega, \text { and } u=0 \text { on } \partial \Omega\right\} .
$$

The pressure $p$ is determined up to an additive constant.

We assume that $\Omega$ has a small and smooth deformation and that the boundary of the deformed domain $\Omega_{\delta}$ is the set of points $\tilde{x}$ defined by

$$
\partial \Omega_{\delta}:=\{\tilde{x}=x+\delta \rho(x) \nu(x), \quad x \in \partial \Omega\}
$$

where $\nu(x)$ is the outward normal vector on on $\partial \Omega$ and $\rho(x)$ is a real function in $C^{2}(\partial \Omega)$ that satisfies

$$
\|\rho(x)\|_{C^{2}(\partial \Omega)}<1
$$

Obviously, the domain $\Omega_{\delta}$ is of class $C^{2}$ and the Dirichlet eigenvalue problem for the Stokes system can be defined in $\Omega_{\delta}$ as well.

In this paper, we derive the asymptotic of eigenvalues, eigenfunctions and the eigenpressures solutions to the Stokes system:

$$
\begin{cases}-\Delta v_{\delta}+\nabla \cdot p_{\delta}=\lambda_{\delta} v_{\delta} & \text { in } \Omega_{\delta} \\ \nabla \cdot v_{\delta}=0 & \text { in } \Omega_{\delta} \\ v_{\delta}=0 & \text { in } \partial \Omega_{\delta} .\end{cases}
$$

Here we suppose that the eigenvalue $\lambda_{0}$ is simple. Then the eigenvalue $\lambda_{\delta}$ is simple and is near to $\lambda_{0}$ associated to the normalized eigenfunction $v_{\delta}$.

To the best of our knowledge, this is the first work to rigorously investigate Stokes eigenvalue problem in the presence of the perturbation (2) and derive high-order terms in the asymptotic expansion of $\lambda_{\delta}-\lambda_{0}$ and $v_{\delta}-v_{0}$ when $\delta \rightarrow 0$. However, by the same method, one can derive asymptotic formula for the Neumann problem as well.

Zuazua and Ortega have proved in [13] the regularity of the eigenvalues and eigenfunctions of the Stokes system with respect to the perturbation parameter, by using the Lyapunov-Schmidt method. Their proofs are essentially inspired in the work of J. Albert [1, 2] for the Laplace operator. Our analysis and uniform asymptotic formulas of eigenvalues and eigenfunctions, which are represented by the single-layer potential involving the Green function, are considerably different from those in [8, 13]. 
This paper is organized as follows. In section 1, we describe the main problem in this work. In section 2, we develop a boundary integral formulation for solving the eigenvalue problem (4), and we present some preliminary results. In the last section, we derive by layer potentials techniques formal asymptotic expansion for both eigenvalues and eigenfunctions of the Stokes operator.

\section{Integral equations method}

We now develop a boundary integral formulation for solving the eigenvalue problems (11) and (41). The components of the fundamental Stokes tensor $\Gamma$ and those of the associated pressure vector $P$, which determine the fundamental solution $(\Gamma, P)$ of the Stokes system in $\mathbb{R}^{3}$, are given by (see for instance [1] )

$$
\left\{\begin{array}{l}
\Gamma_{i j}(\lambda, x)=\frac{1}{4 \pi} \frac{\delta_{i j} e^{i \lambda|x|}}{|x|}+\frac{1}{4 \pi \lambda^{2}} \partial_{x_{i}} \partial_{x_{j}}\left(\frac{e^{i \lambda|x|}-1}{|x|^{3}}\right) \\
P_{i}(x)=\frac{1}{4 \pi} \frac{x_{i}}{|x|^{3}} .
\end{array}\right.
$$

We recall that the ith row, $\Gamma_{i}$ of $\Gamma$ satisfies

$$
\begin{cases}-\Delta \Gamma_{i}+\nabla \cdot P_{i}(x)-\lambda \Gamma_{i}=e^{i} \delta(x) & \text { in } \mathbb{R}^{3} \\ \nabla \cdot \Gamma_{i}=0 & \text { in } \mathbb{R}^{3}\end{cases}
$$

where $\left(e^{i} ; i=1,2,3\right)$ is the orthonormal basis of $\mathbb{R}^{3}$.

\subsection{The potential theory for the Stokes system}

Let us denote by $\varphi=\left(\varphi_{1}, \varphi_{2}, \varphi_{3}\right)$ a complex vector-valued function in the class $C^{0}(\partial \Omega)$.

The hydrodynamic single layer potential with density $\varphi \in C^{0}(\partial \Omega)^{3}$ is the vector function $\mathcal{S}(\lambda) \varphi(x)$ defined by

$$
\mathcal{S}(\lambda) \varphi(x):=\int_{\partial \Omega} \Gamma(\lambda,|x-y|) \varphi(y) d \sigma(y), \quad x \in \mathbb{R}^{3} \backslash \partial \Omega .
$$

The pressure term $\mathcal{Q}$ corresponding to the single layer potential is the function given by

$$
\mathcal{Q} \varphi(x):=\int_{\partial \Omega} P(x, y) \varphi(y) d \sigma(y), \quad x \in \mathbb{R}^{3} \backslash \partial \Omega .
$$

For a cureful stady of these potentials, one can refer to [11], [17], [10].

Taking into account the well known properties of Green function $\Gamma$, one obtains the result that the pair $(\mathcal{S} \varphi, \mathcal{Q} \varphi)$ are smooth functions in each of the domains $\Omega$ and $\mathbb{R}^{3} \backslash \bar{\Omega}$ respectively. Also these functions are classical solution to the Stokes system (1).

The continuity and jump relations of the Stokes surface potentials on the boundary $\partial \Omega$ are described in the following proposition (see [6] pp. 41-42 or [17] p. 66 ):

Proposition 2.1 Let $\varphi \in C^{0}(\partial \Omega)^{3}$ and let $\mathcal{S}$ denotes the surface potential defined in (7). Then on the boundary $\partial \Omega$ the following continuity and jump relations are satisfied:

$$
\begin{gathered}
\left.(\mathcal{S}(\lambda) \varphi)\right|_{+}=\left.(\mathcal{S}(\lambda) \varphi)\right|_{-}=\mathcal{S}(\lambda) \varphi \\
\left.\frac{\partial \mathcal{S}(\lambda)(\varphi)}{\partial \nu}(x)\right|_{ \pm}= \pm \frac{\varphi(x)}{2}+\int_{\partial \Omega} \frac{\partial \Gamma(\lambda,|x-y|)}{\partial \nu(x)} \varphi(y) d \sigma(y) .
\end{gathered}
$$




\subsection{The boundary integral formulation of the problem}

In this section, we give a boundary integral formulation in order to solve the eigenvalue problems (11) and (41).

Proposition 2.2 Suppose $\rho$ satisfies (3). Then, there exists $\delta_{0}>0$ such that the map $\Psi_{\delta}(x)$ defined by

$$
\Psi_{\delta}(x)=x+\delta \rho(x) \nu(x)
$$

is a $C^{2}$ - diffeomorphism from $\partial \Omega$ to $\partial \Omega_{\delta}$ for $\delta<\delta_{0}$. In addition, the following equality holds

$$
\operatorname{det}\left(\nabla \Psi_{\delta}\right)=1+\operatorname{tr} \nabla(\rho \nu) \delta+\frac{1}{2}\left[(\operatorname{tr} \nabla(\rho \nu))^{2}-\operatorname{tr}\left(\nabla(\rho \nu)^{2}\right)\right] \delta^{2}+\operatorname{det}(\nabla(\rho \nu)) \delta^{3},(9)
$$

where $\operatorname{tr}$ means the trace of a matrix. Moreover, we have $\operatorname{tr} \nabla(\rho \nu)=\operatorname{div}(\rho \nu)$.

Proof. Recall that $\partial \Omega$ is $C^{3}$ and so $\nu(x)$ is a $C^{2}$ vector-valued function. Since the function $\rho(x)$ is $C^{2}$, the map $\Psi_{\delta}(x)$ is also $C^{2}$. A simple calculation yields the equality (9). Consequently, for $\delta$ small enough the map $\Psi_{\delta}(x)$ is a $C^{2}$ - diffeomorphism from $\partial \Omega$ to $\partial \Omega_{\delta}$.

We further denote $\Psi_{\delta}^{-1}$ the reciproque function of $\Psi_{\delta}(x)$. Thanks to $\Psi_{\delta}^{-1}$, we can define the operator $\mathcal{A}_{\delta}(\lambda)$ as follows:

$$
\mathcal{A}_{\delta}(\lambda) \varphi(x)=\left(\mathcal{S}_{\Omega_{\delta}}(\lambda) \varphi\left(\Psi_{\delta}^{-1}\right)\right)\left(\Psi_{\delta}(x)\right), \quad \varphi \in\left(L^{2}(\partial \Omega)\right)^{3}
$$

where $\mathcal{S}_{\Omega_{\delta}}(\lambda)$ is the hydrodynamic single layer potential given by (7) when we have replaced the boundary $\partial \Omega$ by $\partial \Omega_{\delta}$.

For $i, j \in\{1,2,3\}$, we can define the $j^{\text {th }}$-component of the vector-valued function $\mathcal{A}_{\delta}(\lambda)$ as follows:

$$
\left(\mathcal{A}^{\delta}(\lambda) \varphi\right)_{j}(x)=\left(\left(\mathcal{S}_{\Omega_{\delta}}(\lambda) \varphi\right)_{j}\left(\Psi_{\delta}^{-1}\right)\right)\left(\Psi_{\delta}(x)\right), \quad \varphi \in\left(L^{2}(\partial \Omega)\right)^{3} .
$$

The $j^{t h}$-component of the single layer potential $\mathcal{S}_{\Omega_{\delta}}(\lambda)$ is given by

$$
\left(\mathcal{S}_{\Omega_{\delta}}(\lambda) \varphi\right)_{j}(\tilde{x}):=\int_{\partial \Omega_{\delta}} \Gamma_{j i}(\lambda,|\tilde{x}-\tilde{y}|) \varphi_{i}(\tilde{y}) d \sigma_{\delta}(\tilde{y}), \quad \tilde{x} \in \mathbb{R}^{3} \backslash \partial \Omega_{\delta}, j=1,2,3,
$$

where $\varphi_{i}$ is the $i^{t h}$-component of the vector-valued function $\varphi$. Using Proposition 2.2. relations (11)-(12) and the continuity relations given by Proposition 2.1, we obtain for $x \in \partial \Omega$ that

$$
\left(\mathcal{A}^{\delta}(\lambda) \varphi\right)_{j}(x)=\int_{\partial \Omega} \Gamma_{j i}\left(\lambda,\left|\Psi_{\delta}(x)-\Psi_{\delta}(y)\right|\right) \operatorname{det}\left(\nabla \Psi_{\delta}(y)\right) \varphi_{i}(y) d \sigma(y), \quad j=1,2,3 .
$$

Let $\mathcal{A}_{0}$ the operator defined as in (10) by

$$
\mathcal{A}_{0} \phi=\mathcal{S}(\lambda) \phi
$$

where $\phi \in\left(L^{2}(\partial \Omega)\right)^{3}$. Then, we have the following result, which is a slight variation of the Lemma 6.1 due to Ammari and Triki [3] on the scalar eigenvalue problem for the Laplacian. 
Proposition 2.3 The operator-valued function $\mathcal{A}_{0}(\lambda): H^{-1 / 2}(\partial \Omega)^{3} \rightarrow H^{1 / 2}(\partial \Omega)^{3}$ is Fredholm of index zero in $\mathbb{C} \backslash i \mathbb{R}^{-}$. In addition the Dirichlet eigenvalues of the Stokes system (1) are exactly its real zeros.

From Proposition 2.3 we know that if $\lambda_{0}$ is an eigenvalue of (1) then $\lambda_{0}$ is a real zero of $\mathcal{A}_{0}(\lambda)$. Moreover, for $\epsilon_{0}$ small enough, the function $\mathcal{A}_{0}^{-1}(\lambda)$ is meromorphic in $\overline{D_{\epsilon_{0}}}\left(\lambda_{0}\right)$, where $D_{\epsilon_{0}}\left(\lambda_{0}\right)$ the disc of center $\lambda_{0}$ and radius $\epsilon_{0}$, and $\lambda_{0}$ is its unique pole in $\overline{D_{\epsilon_{0}}}$. Furthermore we have the Laurent expansion

$$
\mathcal{A}_{0}^{-1}(\lambda)=\left(\lambda-\lambda_{0}\right)^{-1} \ell_{0}+R_{0}(\lambda),
$$

where $\ell_{0}: \operatorname{Ker} \mathcal{A}_{0}\left(\lambda_{0}\right) \rightarrow \operatorname{Ker} \mathcal{A}_{0}\left(\lambda_{0}\right)$, and $R_{0}(\lambda)$ is a holomorphic function.

Our main results in this section are summarized in the following theorem.

Theorem 2.1 Suppose that the eigenvalue $\lambda_{0}$ of (1) is with multiplicity 1. Then, there exists a positive constant $\delta_{0}\left(\epsilon_{0}\right)$ such that for $|\delta|<\delta_{0}$, the operator-valued function $\lambda \mapsto \mathcal{A}_{\delta}(\lambda)$ has a real zero $\lambda(\delta)$ in $\overline{D_{\epsilon_{0}}}\left(\lambda_{0}\right)$. This zero is exactly the eigenvalue of the perturbed eigenvalue problem (4), and is an analytic function with respect to $\delta$ in ]$-\delta_{0}, \delta_{0}\left[\right.$. It satisfies $\lambda(0)=\lambda_{0}$. Moreover, the following assertions hold:

$$
\begin{aligned}
& \mathcal{A}_{\delta}^{-1}(\lambda)=(\lambda-\lambda(\delta))^{-1} \ell(\delta)+R_{\delta}(\lambda), \\
& \ell(\delta): \operatorname{Ker}\left(\mathcal { A } _ { \delta } ( \lambda ( \delta ) ) \rightarrow \operatorname { K e r } \left(\mathcal{A}_{\delta}(\lambda(\delta)),\right.\right.
\end{aligned}
$$

where $R_{\delta}(\lambda)$ is a holomorphic function with respect to $\left.(\delta, \lambda) \in\right]-\delta_{0}, \delta_{0}\left[\times D_{\epsilon_{0}}\left(\lambda_{0}\right)\right.$.

\section{Asymptotic behaviors}

\subsection{High-order terms in the expansion of $\mathcal{A}_{\delta}$}

We now present some basic results related to shape perturbation. To begin, let $\left(\tau_{1}(x), \tau_{2}(x)\right)$ be the orthornormal basis of the tangent plan to the surface $\partial \Omega$ at a regular point $x$. Their cross product is so orthogonal to $\partial \Omega$ at the point $x$. By changing their order, we can assume that $\tau_{1} \times \tau_{2}$ is a vector pointing towards the exterior of the surface $\partial \Omega$. Then dividing it by its length yields the unit normal vector $\nu(x)$, that is

$$
\nu_{0}(x)=\frac{\tau_{1}(x) \times \tau_{2}(x)}{\left|\tau_{1}(x) \times \tau_{2}(x)\right|},
$$

for $x \in \partial \Omega$. Evidently $\nu_{0}=\nu$, where $\nu$ was introduced in section 1 .

Set

$$
\tau_{1}^{\delta}=\operatorname{grad} \Psi_{\delta} \cdot \tau_{1}, \quad \text { and } \tau_{2}^{\delta}=\operatorname{grad} \Psi_{\delta} \cdot \tau_{2} .
$$

Using Proposition 2.2, we find that

$$
\tau_{1}^{\delta}=\tau_{1}+\delta M \tau_{1}, \quad \text { and } \tau_{2}^{\delta}=\tau_{2}+\delta M \tau_{2},
$$

where the $(3 \times 3)$ - matrix $M$ is given by

$$
M=\left(\begin{array}{lll}
\partial_{1}\left(\rho \nu_{1}\right) & \partial_{2}\left(\rho \nu_{1}\right) & \partial_{3}\left(\rho \nu_{1}\right) \\
\partial_{1}\left(\rho \nu_{2}\right) & \partial_{2}\left(\rho \nu_{2}\right) & \partial_{3}\left(\rho \nu_{2}\right) \\
\partial_{1}\left(\rho \nu_{3}\right) & \partial_{2}\left(\rho \nu_{3}\right) & \partial_{3}\left(\rho \nu_{3}\right)
\end{array}\right)
$$


with $\nu_{i}$ means the $i$-th $(i=1,2,3)$ component of the vector $\nu$.

For $\delta$ sufficiently small, one can see that the outward unit normal vector to $\partial \Omega_{\delta}$ is given by

$$
\nu_{\delta}(x)=\frac{\tau_{1}^{\delta}(x) \times \tau_{2}^{\delta}(x)}{\left|\tau_{1}^{\delta}(x) \times \tau_{2}^{\delta}(x)\right|}
$$

for $x \in \partial \Omega$. Then, the following asymptotic expansion holds.

Proposition 3.1 Let $\nu_{0}$ be given by (16). Then, the outward unit normal $\nu_{\delta}(x)$ to $\partial \Omega_{\delta}$ at $x$, can be expanded uniformly as

$$
\nu_{\delta}(x)=\nu^{(0)}(x)+\sum_{n=1}^{\infty} \delta^{n} \nu^{(n)}(x), \quad x \in \partial \Omega,
$$

where the vector-valued functions $\nu^{(n)}$ are uniformly bounded regardless of $n$. In particular, for $x \in \partial \Omega$

$$
\begin{gathered}
\nu^{(0)}(x)=\nu(x) \\
\nu^{(1)}=\frac{1}{\left|\tau_{1} \times \tau_{2}\right|}\left[\tau_{1} \times M \tau_{2}+M \tau_{1} \times \tau_{2}-\left(\nu_{0} \cdot\left(\tau_{1} \times M \tau_{2}+M \tau_{1} \times \tau_{2}\right)\right) \nu_{0}\right] .
\end{gathered}
$$

Proof.

Considering the expansions (17) for $\delta$ sufficiently small, the relation (18) becomes

$$
\nu_{\delta}=\frac{\mathbf{a}+\delta \mathbf{b}+\delta^{2} \mathbf{c}}{\left|\mathbf{a}+\delta \mathbf{b}+\delta^{2} \mathbf{c}\right|}
$$

where $\mathbf{a}, \mathbf{b}$, and $\mathbf{c}$ are vector-valued functions given by

$$
\begin{array}{r}
\mathbf{a}=\tau_{1} \times \tau_{2}, \\
\mathbf{b}=M \tau_{1} \times \tau_{2}+\tau_{1} \times M \tau_{2}, \\
\mathbf{c}=M \tau_{1} \times M \tau_{2} .
\end{array}
$$

So that, by expanding the quotient (22) as $\delta$ tends to zero, we get the desired results.

Next, one can use Proposition 2.2 to get a uniformly convergent expansion for the surface element as follows:

Proposition 3.2 Let $\tilde{y}=\Psi_{\delta}(y)$ where $\Psi_{\delta}(y)$ is given by (8) for $y \in \partial \Omega$. Then, the following expansion for the surface element $d \sigma_{\delta}(\tilde{y})$ holds uniformly for $y \in \partial \Omega$ :

$$
d \sigma_{\delta}(\tilde{y})=\operatorname{det}\left(\nabla \Psi_{\delta}\right) d \sigma(y)=\left(\sigma_{0}(y)+\sigma_{1}(y) \delta+\sigma_{2}(y) \delta^{2}+\sigma_{3}(y) \delta^{3}\right) d \sigma(y),
$$

where $\sigma_{0} \equiv 1, \sigma_{1}(y)=\nabla \cdot(\rho \nu), \sigma_{2}(y)=\frac{1}{2}\left[(\operatorname{tr} \nabla(\rho \nu))^{2}-\operatorname{tr}\left(\nabla(\rho \nu)^{2}\right)\right]$, and $\sigma_{3}(y)=$ $\operatorname{det}(\nabla(\rho \nu))$. 
Set

$$
\begin{array}{ll}
\tilde{x}=\Psi_{\delta}(x), & x \in \partial \Omega \\
\tilde{y}=\Psi_{\delta}(y), & y \in \partial \Omega,
\end{array}
$$

and define

$$
\Theta(x, y):=\frac{1}{\delta}\left(\Psi_{\delta}(x)-\Psi_{\delta}(y)-(x-y)\right) \text { for } \delta \neq 0 .
$$

Recall that $\Psi_{\delta}$ is a $C^{2}$ vector-valued function on $\partial \Omega$, then $\Theta$ is also a $C^{2}$ function on $(\partial \Omega)^{2}$. Moreover, the following holds.

Proposition 3.3 The vector-valued function $\Theta(x, y)$ is $C^{2}$ on $(\partial \Omega)^{2}$ and there exists a constant $C>0$ that only depends on $\Omega$ and $\rho$ such that

$$
\begin{array}{r}
|\Theta(x, y)| \leq C|x-y|, \\
|\Theta(x, y) \cdot(x-y)| \leq C|x-y|^{2},
\end{array}
$$

for all $x, y \in \partial \Omega$.

Proof. Expression (26) shows that

$$
\Theta(x, y)=\rho(x) \nu(x)-\rho(y) \nu(y) .
$$

Since $\partial \Omega$ is a $C^{3}$ surface, there exists a constant $C^{\prime}>0$ such that

$$
\begin{array}{r}
|\nu(x)-\nu(y)| \leq C^{\prime}|x-y|, \\
|\nu(x) \cdot(x-y)| \leq C^{\prime}|x-y|^{2},
\end{array}
$$

for all $x, y \in \partial \Omega$.

The last inequalities and the $C^{2}$ regularity of $\rho$ yield the results of the proposition.

Now, by using (26) we get

$$
\tilde{x}-\tilde{y}=x-y+\delta \Theta(x, y), \quad(x, y) \in \partial \Omega \times \partial \Omega,
$$

and the following results hold.

Lemma 3.1 Let $r>0$ be a fixed real and $m \geq 2$ be a fixed integer. The following asymptotic expansions

$$
\begin{array}{r}
\frac{e^{i \lambda|\tilde{x}-\tilde{y}|}}{|\tilde{x}-\tilde{y}|^{m}}=\frac{e^{i \lambda|x-y|}}{|x-y|^{m}}\left(1+\delta T_{1}^{(m)}(x, y)+\sum_{n \geq 2} \delta^{n} T_{n}^{(m)}(\lambda ; x, y)\right), \\
\partial_{\tilde{x}_{i}} \partial_{\tilde{x}_{j}} \frac{e^{i \lambda|\tilde{x}-\tilde{y}|}-1}{|\tilde{x}-\tilde{y}|^{3}}=R_{0}^{(i j)}(\lambda ; x, y)+\delta R_{1}^{(i j)}(\lambda ; x, y)+\sum_{n \geq 2} \delta^{n} R_{n}^{(i j)}(\lambda ; x, y)
\end{array}
$$

hold uniformly for $(\lambda, x, y) \in B_{r}(0) \times \partial \Omega^{2}$, where $B_{r}(0)$ is a ball in the complex plane of center zero and radius $r$. In addition the functions $T_{n}^{(m)}(\lambda ; x, y)$ and $R_{n}^{(i j)}(\lambda ; x, y)$ are smooth and bounded uniformly on $B_{r}(0) \times \partial \Omega^{2}$. The first coefficients are given by:

$$
R_{0}^{(i j)}(\lambda ; x, y):=\partial_{x_{i}} \partial_{x_{j}} \frac{e^{i \lambda|x-y|}-1}{|x-y|^{3}}
$$


and

$$
\begin{gathered}
R_{1}^{(i j)}(\lambda ; x, y)=(x-y) \cdot \Theta(x, y)\left(-\lambda^{2} \delta_{i j}-3 i \lambda \frac{\delta_{i j}}{r}-i \lambda^{3} \frac{r_{i} r_{j}}{r}+7 \lambda^{2} \frac{r_{i} r_{j}}{r^{2}}+15 i \lambda \frac{r_{i} r_{j}}{r^{3}}\right) \frac{e^{i \lambda r}}{r^{5}} \\
+(x-y) \cdot \Theta(x, y)\left(4 i \lambda \frac{\delta_{i j}}{r}-15 \frac{\delta_{i j}}{r^{2}}-\lambda^{2}\left[-5 \frac{r_{i} r_{j}}{r^{2}}+\frac{\left(r_{i} \theta_{j}+r_{j} \theta_{i}\right)}{(x-y) \cdot \Theta(x, y)}-7 i \lambda\left[-6 \frac{r_{i} r_{j}}{r^{3}}+\frac{1}{r} \frac{\left(r_{i} \theta_{j}+r_{j} \theta_{i}\right)}{(x-y) \cdot \Theta(x, y)}\right]\right.\right. \\
+15\left[-7 \frac{r_{i} r_{j}}{r^{4}}+\frac{1}{r^{2}} \frac{\left(r_{i} \theta_{j}+r_{j} \theta_{i}\right)}{(x-y) \cdot \Theta(x, y)}\right) \frac{e^{i \lambda r}}{r^{5}} \\
+15 \frac{(x-y) \cdot \Theta(x, y)}{r^{7}}\left(\delta_{i j}-\left[-7 \frac{r_{i} r_{j}}{r^{2}}+\frac{\left(r_{i} \theta_{j}+r_{j} \theta_{i}\right)}{(x-y) \cdot \Theta(x, y)}\right]\right),
\end{gathered}
$$

where $r=|x-y|, r_{j}=x_{j}-y_{j}, \theta_{j}$ means the $j^{\text {th }}$ component of $\Theta$, and $\delta_{i j}$ means the Kronecker index.

Proof. For $m=2$ and $x \neq y$ we have

$$
|\tilde{x}-\tilde{y}|^{2}=|x-y+\delta \Theta(x, y)|^{2}=|x-y|^{2}\left(1+\delta c_{1}^{(2)}(x, y)+\delta^{2} c_{2}^{(2)}(x, y)\right),
$$

where

$$
c_{1}^{(2)}(x, y)=\frac{2 \Theta(x, y) \cdot(x-y)}{|x-y|^{2}}, \quad c_{2}^{(2)}(x, y)=\frac{\Theta(x, y) \cdot \Theta(x, y)}{|x-y|^{2}}
$$

Proposition 3.1 shows that $c_{1}^{(2)}(x, y)$ and $c_{2}^{(2)}(x, y)$ are bounded uniformly on $\partial \Omega^{2}$.

For $m>2$, we have:

$$
|\tilde{x}-\tilde{y}|^{m}=|x-y+\delta \Theta(x, y)|^{m}=|x-y|^{m}\left|1+\delta \frac{\Theta(x, y)}{|x-y|}\right|^{m},
$$

where $x \neq y$.

Using (26), Proposition 2.2 and Proposition 3.3 , we see that the regular vector-valued function $(x, y) \mapsto \frac{\Theta(x, y)}{|x-y|}$ is well defined on $\partial \Omega \times \partial \Omega$, and it is independent of $\delta$.

Therefore, we can expand

$$
|\tilde{x}-\tilde{y}|^{m}=c_{0}^{(m)}(x, y)+\sum_{n=1}^{\infty} \delta^{n} c_{n}^{(m)}(x, y) \quad \text { uniformly on } \partial \Omega \times \partial \Omega,
$$

where the first coefficients $c_{0}^{(m)}(x, y)=|x-y|^{m}, c_{1}^{(m)}(x, y)=m<x-y, \Theta(x, y)>$ $|x-y|^{m-2}$ and $c_{2}^{(m)}(x, y)=\frac{m}{2}|x-y|^{m}\left[\left|\frac{\Theta(x, y)}{|x-y|}\right|^{2}+(m-2)\left(<\frac{x-y}{|x-y|^{2}}, \frac{\Theta(x, y)}{|x-y|}>\right)^{2}\right]$. Moreover, if $m$ is even, then $c_{n}^{(m)}(x, y)=0$ for $n \geq m+1$.

Now combining (33) for $m=1$ with the well known asymptotic expansion of the exponential function, we immediately get

$$
e^{i \lambda|\tilde{x}-\tilde{y}|}=\sum_{n=0}^{\infty} \delta^{n} K_{n}(\lambda ; x, y) \quad \text { uniformly on } B_{r}(0) \times \partial \Omega \times \partial \Omega,
$$


where the first coefficients $K_{0}(\lambda ; x, y)=e^{i \lambda c_{0}^{(1)}}=e^{i \lambda|x-y|}$, and the coefficients $K_{n}(\lambda ; x, y)$ can be deduced recursively from $c_{n}^{(1)}$.

Thanks to relations (30), (33) and (34), we can obtain the desired result given by (31) where the coefficients $T_{n}^{(m)}$ can be deduced easily from $c_{n}^{(m)}$ and $K_{n}$.

To prove relation (32), we firstly expand

$$
\begin{gathered}
\partial_{\tilde{x}_{i}} \partial_{\tilde{x}_{j}} \frac{e^{i \lambda|\tilde{x}-\tilde{y}|}-1}{|\tilde{x}-\tilde{y}|^{3}}=\frac{1}{|\tilde{x}-\tilde{y}|^{3}} \partial_{\tilde{x}_{i}} \partial_{\tilde{x}_{j}}\left(e^{i \lambda|\tilde{x}-\tilde{y}|}-1\right)+\partial_{\tilde{x}_{i}}\left(\frac{1}{|\tilde{x}-\tilde{y}|^{3}}\right) \partial_{\tilde{x}_{j}}\left(e^{i \lambda|\tilde{x}-\tilde{y}|}-1\right) \\
+\partial_{\tilde{x}_{j}}\left(\frac{1}{|\tilde{x}-\tilde{y}|^{3}}\right) \partial_{\tilde{x}_{i}}\left(e^{i \lambda|\tilde{x}-\tilde{y}|}-1\right)+\partial_{\tilde{x}_{i}} \partial_{\tilde{x}_{j}}\left(\frac{1}{|\tilde{x}-\tilde{y}|^{3}}\right)\left(e^{i \lambda|\tilde{x}-\tilde{y}|}-1\right) .
\end{gathered}
$$

To simplify, let us denote $r=|x-y|, r_{i}=x_{i}-y_{i}, \tilde{r}=|\tilde{x}-\tilde{y}|$, and $\hat{r}_{j}$ defined by the relation

$$
\partial_{\tilde{x}_{j}} \tilde{r}=\frac{\hat{r}_{j}}{\tilde{r}} .
$$

Then,

$$
\partial_{\tilde{x}_{j}}\left(\frac{1}{\tilde{r}^{3}}\right)=-3 \frac{\hat{r}_{j}}{\tilde{r}^{5}}, \quad \text { and } \partial_{\tilde{x}_{i}} \partial_{\tilde{x}_{j}}\left(\frac{1}{\tilde{r}^{3}}\right)=-3 \frac{\delta_{i j}}{\tilde{r}^{5}}+15 \frac{\hat{r}_{i} \hat{r}_{j}}{\tilde{r}^{7}} .
$$

Now, by using the following result

$$
\partial_{\tilde{x}_{j}}\left(e^{i \lambda|\tilde{x}-\tilde{y}|}-1\right)=\partial_{\tilde{x}_{j}}(i \lambda \tilde{r}) e^{i \lambda \tilde{r}}=i \lambda \frac{\hat{r}_{j}}{\tilde{r}} e^{i \lambda \tilde{r}}
$$

we get that

$$
\partial_{\tilde{x}_{i}} \partial_{\tilde{x}_{j}}\left(e^{i \lambda|\tilde{x}-\tilde{y}|}-1\right)=i \lambda\left[\partial_{\tilde{x}_{i}}\left(\frac{\hat{r}_{j}}{\tilde{r}}\right) e^{i \lambda \tilde{r}}+\frac{\hat{r}_{j}}{\tilde{r}} \partial_{\tilde{x}_{i}} e^{i \lambda \tilde{r}}\right]=i \lambda\left[\frac{\delta_{i j}}{\tilde{r}}-\frac{\hat{r}_{i} \hat{r}_{j}}{\tilde{r}^{3}}+i \lambda \frac{\hat{r}_{i} \hat{r}_{j}}{\tilde{r}^{2}}\right] e^{i \lambda \tilde{r}} .
$$

To find the desired result in (32), we may use (37), (38) and (39) to see that the relation (35) verifies:

$$
\partial_{\tilde{x}_{i}} \partial_{\tilde{x}_{j}} \frac{e^{i \lambda|\tilde{x}-\tilde{y}|}-1}{|\tilde{x}-\tilde{y}|^{3}}=\left[i \lambda \frac{\delta_{i j}}{\tilde{r}^{4}}-3 \frac{\delta_{i j}}{\tilde{r}^{5}}-\lambda^{2} \frac{\hat{r}_{i} \hat{r}_{j}}{\tilde{r}^{5}}-7 i \lambda \frac{\hat{r}_{i} \hat{r}_{j}}{\tilde{r}^{6}}+15 \frac{\hat{r}_{i} \hat{r}_{j}}{\tilde{r}^{7}}\right] e^{i \lambda \tilde{r}}+3 \frac{\delta_{i j}}{\tilde{r}^{5}}-15 \frac{\hat{r}_{i} \hat{r}_{j}}{\tilde{r}^{7}} .
$$

On the other hand, the components of the vectorial relation (30) can be given as follows

$$
\tilde{x}_{i}-\tilde{y}_{i}=x_{i}-y_{i}+\delta \theta_{i}(x, y) . \quad i=1,2,3
$$

where $\theta_{i}(x, y)$ means the $i^{\text {th }}$ component of the vector-valued function $\Theta(x, y)$. Then, by relations (36) and (41) we deduce that

$$
\hat{r}_{j}=\tilde{x}_{i}-\tilde{y}_{i}=x_{i}-y_{i}+\delta \theta_{i}(x, y)=r_{i}+\delta \theta_{i}(x, y), \quad i=1,2,3 .
$$

Using both relations (30) and (42), we get the following expansion

$$
\hat{r}_{i} \hat{r}_{j}=\alpha_{0}^{(i j)}+\alpha_{1}^{(i j)} \delta+\alpha_{2}^{(i j)} \delta^{2}
$$

where the first coefficients: $\alpha_{0}^{(i j)}=r_{i} r_{j}, \alpha_{1}^{(i j)}=r_{i} \theta_{j}+r_{j} \theta_{i}$ and $\alpha_{2}^{(i j)}=\theta_{i} \cdot \theta_{j}$. 
Now regarding (33) and using the fact that $c_{0}^{(m)} \neq 0$ for each integer $m$. Then one can expand

$$
\frac{1}{\tilde{r}^{m}}=\kappa_{0}^{(m)}(x, y)+\sum_{n=1}^{\infty} \delta^{n} \kappa_{n}^{(m)}(x, y) \quad \text { uniformly }
$$

where the first coefficients: $\kappa_{0}^{(m)}(x, y)=\left(c_{0}^{(m)}\right)^{-1}(x, y)$, and $\kappa_{1}^{(m)}(x, y)=-\left(c_{1}^{(m)}\left(c_{0}^{(m)}\right)^{-2}\right)(x, y)$. By using (43)-(44), we get that

$$
\frac{\hat{r}_{i} \hat{r}_{j}}{\tilde{r}^{m}}=\beta_{0, m}^{(i j)}+\delta \beta_{1, m}^{(i j)}+\sum_{n=2}^{\infty} \delta^{n} \beta_{n, m}^{(i j)},
$$

where the first coefficients: $\beta_{0, m}^{(i j)}(x, y)=\alpha_{0}^{(i j)}\left(c_{0}^{(m)}\right)^{-1}(x, y)$, and $\beta_{1, m}^{(i j)}(x, y)=-\alpha_{0}^{(i j)}\left(c_{1}^{(m)}\left(c_{0}^{(m)}\right)^{-2}\right)(x, y)+\alpha_{1}^{(i j)}\left(c_{0}^{(m)}\right)^{-1}(x, y)$.

To achieve the proof, we may insert all expansions (34), (44) (for $m \in\{4,5\}$ ), and (45) (for $m \in\{5,6,7\}$ into (40). We get that

$$
\partial_{\tilde{x}_{i}} \partial_{\tilde{x}_{j}} \frac{e^{i \lambda|\tilde{x}-\tilde{y}|}-1}{|\tilde{x}-\tilde{y}|^{3}}=R_{0}^{(i j)}(x, y)+\delta R_{1}^{(i j)}(x, y)+\sum_{n=2}^{\infty} \delta^{n} R_{n}^{(i j)}(x, y)
$$

where the first coefficient:

$$
\begin{gathered}
R_{0}^{(i j)}(\lambda ; x, y)=\left(i \lambda \delta_{i j} \kappa_{0}^{(4)}-3 \delta_{i j} \kappa_{0}^{(5)}-\lambda^{2} \beta_{0,5}^{(i j)}-7 i \lambda \beta_{0,6}^{(i j)}+15 \beta_{0,7}^{(i j)}\right) K_{0}+3 \delta_{i j} \kappa_{0}^{(5)}-15 \beta_{0,7}^{(i j)} \\
=\left[i \lambda \delta_{i j}\left(c_{0}^{(4)}\right)^{-1}-3 \delta_{i j}\left(c_{0}^{(5)}\right)^{-1}-\lambda^{2} r_{i} r_{j}\left(c_{0}^{(5)}\right)^{-1}-7 i \lambda r_{i} r_{j}\left(c_{0}^{(6)}\right)^{-1}+15 r_{i} r_{j}\left(c_{0}^{(7)}\right)^{-1}\right] e^{i \lambda c_{0}^{(1)}} \\
+3 \delta_{i j}\left(c_{0}^{(5)}\right)^{-1}-15 r_{i} r_{j}\left(c_{0}^{(7)}\right)^{-1} .
\end{gathered}
$$

Using the fact that $c_{0}^{(m)}=|x-y|^{m}$, we find that

$$
R_{0}^{(i j)}(\lambda ; x, y):=\partial_{x_{i}} \partial_{x_{j}} \frac{e^{i \lambda|x-y|}-1}{|x-y|^{3}} .
$$

Based on (40), we find that

$$
\begin{gathered}
R_{1}^{(i j)}(\lambda ; x, y)=\left(i \lambda \delta_{i j} \kappa_{0}^{(4)}-3 \delta_{i j} \kappa_{0}^{(5)}-\lambda^{2} \beta_{0,5}^{(i j)}-7 i \lambda \beta_{0,6}^{(i j)}+15 \beta_{0,7}^{(i j)}\right) K_{1} \\
+\left(i \lambda \delta_{i j} \kappa_{1}^{(4)}-3 \delta_{i j} \kappa_{1}^{(5)}-\lambda^{2} \beta_{1,5}^{(i j)}-7 i \lambda \beta_{1,6}^{(i j)}+15 \beta_{1,7}^{(i j)}\right) K_{0}+3 \delta_{i j} \kappa_{1}^{(5)}-15 \beta_{1,7}^{(i j)} .
\end{gathered}
$$

So that, by using the fact that $K_{1}=i \lambda \Theta \cdot \frac{(x-y)}{r} e^{i \lambda c_{0}^{(1)}}$, we get

$$
\begin{aligned}
& R_{1}^{(i j)}(\lambda ; x, y)=i \lambda \Theta \cdot \frac{(x-y)}{r}\left(i \lambda \delta_{i j}\left(c_{0}^{(4)}\right)^{-1}-3 \delta_{i j}\left(c_{0}^{(5)}\right)^{-1}-\lambda^{2} r_{i} r_{j}\left(c_{0}^{(5)}\right)^{-1}-7 i \lambda r_{i} r_{j}\left(c_{0}^{(6)}\right)^{-1}\right. \\
& \left.+15 r_{i} r_{j}\left(c_{0}^{(7)}\right)^{-1}\right) e^{i \lambda c_{0}^{(1)}} \\
& \quad+\left(i \lambda \delta_{i j}\left(c_{0}^{(4)}\right)^{-2} c_{1}^{(4)}-3 \delta_{i j}\left(c_{0}^{(5)}\right)^{-2} c_{1}^{(5)}-\lambda^{2}\left[-r_{i} r_{j}\left(c_{0}^{(5)}\right)^{-2} c_{1}^{(5)}+\left(r_{i} \theta_{j}+r_{j} \theta_{i}\right)\left(c_{0}^{(5)}\right)^{-1}\right]\right. \\
& \left.-7 i \lambda\left[-r_{i} r_{j}\left(c_{0}^{(6)}\right)^{-2} c_{1}^{(6)}+\left(r_{i} \theta_{j}+r_{j} \theta_{i}\right)\left(c_{0}^{(6)}\right)^{-1}\right]+15\left[-r_{i} r_{j}\left(c_{0}^{(7)}\right)^{-2} c_{1}^{(7)}+\left(r_{i} \theta_{j}+r_{j} \theta_{i}\right)\left(c_{0}^{(7)}\right)^{-1}\right]\right) e^{i \lambda c_{0}^{(1)}}
\end{aligned}
$$




$$
+3 \delta_{i j}\left(c_{0}^{(5)}\right)^{-2} c_{1}^{(5)}-15\left[-r_{i} r_{j}\left(c_{0}^{(7)}\right)^{-2} c_{1}^{(7)}+\left(r_{i} \theta_{j}+r_{j} \theta_{i}\right)\left(c_{0}^{(7)}\right)^{-1}\right] .
$$

Using the explicit forms of the coefficients $c_{0}^{(m)}$ and $c_{1}^{(m)}$ given above, we immediately get the desired expression of $R_{1}^{(i j)}(\lambda ; x, y)$. The other coefficients can be deduced easily by the same manner in terms of $c_{n}^{(m)}$.

Now using (41), we obtain for $i, j=1,2,3$ that:

$$
\left(\tilde{x}_{i}-\tilde{y}_{i}\right)\left(\tilde{x}_{j}-\tilde{y}_{j}\right)=\hat{g}_{0}(x, y)+\delta \hat{g}_{1}(x, y)+\delta^{2} \hat{g}_{2}(x, y)
$$

where

$$
\hat{g}_{0}(x, y)=\left(x_{i}-y_{i}\right)\left(x_{j}-y_{j}\right), \quad \hat{g}_{1}(x, y)=\theta_{i}(x, y)\left(x_{j}-y_{j}\right)+\theta_{j}(x, y)\left(x_{i}-y_{i}\right),
$$

and

$$
\hat{g}_{2}(x, y)=\theta_{i}(x, y) \cdot \theta_{j}(x, y)
$$

Now, by using (23), (33) and (46), we immediately get

$$
\frac{\left(\tilde{x}_{i}-\tilde{y}_{i}\right)\left(\tilde{x}_{j}-\tilde{y}_{j}\right)}{|\tilde{x}-\tilde{y}|^{m}} d \sigma_{\delta}(\tilde{y})=\left(\hat{T}_{0}(x, y)+\delta \hat{T}_{1}(x, y)+\sum_{n \geq 2} \delta^{n} \hat{T}_{n}(x, y)\right) d \sigma(y),
$$

where the Taylor coefficients $\hat{T}_{n}$ can be given explicitly with the aid of $\hat{g}_{0}, \hat{g}_{1}$, and $\hat{g}_{2}$.

Next, the following result holds.

Lemma 3.2 The following uniform expansion holds on $\mathbb{R} \times \partial \Omega \times \partial \Omega$ :

$$
\left(\tilde{x}_{i}-\tilde{y}_{i}\right)\left(\tilde{x}_{j}-\tilde{y}_{j}\right)|\tilde{x}-\tilde{y}| e^{i \lambda|\tilde{x}-\tilde{y}|} d \sigma_{\delta}(\tilde{y})=E_{\delta}(\lambda ; x, y) d \sigma(y)=\sum_{n=0}^{\infty} \delta^{n} E_{n}(\lambda ; x, y) d \sigma(y)
$$

with

$$
E_{0}(\lambda ; x, y)=\hat{g}_{0}(x, y)|x-y| e^{i \lambda|x-y|}
$$

and the other coefficients $E_{n}$ are deduced from those $\sigma_{n}, c_{n}^{(1)}, K_{n}$ and $\hat{g}_{n}$.

Proof. From the proof of Lemma 3.1 (for $m=1$ ) and from relations (23) and (46), one can get that

$$
\begin{gathered}
\left(\tilde{x}_{i}-\tilde{y}_{i}\right)\left(\tilde{x}_{j}-\tilde{y}_{j}\right)|\tilde{x}-\tilde{y}| e^{i \lambda|\tilde{x}-\tilde{y}|} d \sigma_{\delta}(\tilde{y})=\left(\hat{g}_{0}(x, y)+\delta \hat{g}_{1}(x, y)+\right. \\
\left.\delta^{2} \hat{g}_{2}(x, y)\right)\left(\sum_{n=0}^{\infty} \delta^{n} c_{n}^{(1)}\right)\left(\sum_{n=0}^{\infty} K_{n}(\lambda ; x, y)\right)\left(\sigma_{0}(y)+\delta \sigma_{1}(y)+\right. \\
\left.\delta^{2} \sigma_{2}(y)+\delta^{3} \sigma_{3}(y)\right) d \sigma(y) .
\end{gathered}
$$

By collecting terms of equal powers in the above relation, one can deduce easily the uniform expansion (48) with

$$
E_{0}(\lambda ; x, y)=\hat{g}_{0}(x, y)|x-y| e^{i \lambda|x-y|}
$$


Now, from (5) we have

$$
\begin{gathered}
\Gamma_{i j}(\lambda,|x-y|)=\frac{e^{i \lambda r}}{4 \pi}\left[\frac{\delta_{i j}}{r}-\frac{\hat{x}_{i} \hat{x}_{j}}{r^{3}}\right]+\frac{e^{i \lambda r}}{4 \pi \lambda}\left[i \frac{\delta_{i j}}{r^{4}}-4 i \frac{\hat{x}_{i} \hat{x}_{j}}{r^{4}}-3 i r \hat{x}_{i} \hat{x}_{j}\right] \\
+\frac{1}{4 \pi \lambda^{2}}\left[-3 \frac{\delta_{i j}}{r^{5}}+15 \frac{\hat{x}_{i} \hat{x}_{j}}{r^{7}}\right]+\frac{e^{i \lambda r}}{4 \pi \lambda^{2}}\left[-3 \frac{\delta_{i j}}{r^{5}}+15 \frac{\hat{x}_{i} \hat{x}_{j}}{r^{3}}\right],
\end{gathered}
$$

where $r=|x-y|$ and $\hat{x}_{i}=x_{i}-y_{i}$. Then, by inserting relations (31), (46), (47) and that given by Lemma 3.2 into above identity, we immediately get the following main results

Proposition 3.4 Let the perturbed boundary $\partial \Omega_{\delta}$ defined by (2). Let $\tilde{x}$ and $\tilde{y}$ given by (24)-(25), and the surface element $d \sigma_{\delta}(\tilde{y})$ given by (23). Then, the components $\Gamma_{i j}, 1 \leq i, j \leq 3$ of the fundamental Stokes tensor can be expanded uniformly as:

$$
\begin{gathered}
\Gamma_{i j}(\lambda,|\tilde{x}-\tilde{y}|) d \sigma_{\delta}(\tilde{y})=\left(\Gamma_{i j}^{(0)}(\lambda,|x-y|)+\delta \Gamma_{i j}^{(1)}(\lambda,|x-y|)\right. \\
\left.+\sum_{n \geq 2} \delta^{n} \Gamma_{i j}^{(n)}(\lambda,|x-y|)\right) d \sigma(y), \quad y \in \partial \Omega
\end{gathered}
$$

where the first coefficients:

$$
\begin{gathered}
\Gamma_{i j}^{(0)}(\lambda,|x-y|):=\frac{\delta_{i j}}{4 \pi}\left(T_{0}^{(1)}+\frac{i}{\lambda} T_{0}^{(4)}-3 \frac{r_{0}}{\lambda^{2}}-3 \frac{T_{0}^{(5)}}{\lambda^{2}}\right)+\frac{1}{4 \pi}\left(\frac{15}{\lambda^{2}}-1\right) T_{0}^{(3)} \hat{g}_{0}-\frac{4 i}{4 \pi \lambda} T_{0}^{(4)} \hat{g}_{0} \\
-\frac{3 i}{4 \pi \lambda} E_{0}+\frac{15}{4 \pi \lambda^{2}} \hat{T}_{0},
\end{gathered}
$$

and

$$
\begin{aligned}
\Gamma_{i j}^{(1)}(\lambda,|x-y|):= & \frac{\delta_{i j}}{4 \pi}\left(T_{1}^{(1)}+\frac{i}{\lambda} T_{1}^{(4)}-3 \frac{r_{1}}{\lambda^{2}}-3 \frac{T_{1}^{(5)}}{\lambda^{2}}\right)+\frac{1}{4 \pi}\left(\frac{15}{\lambda^{2}}-1\right)\left[T_{0}^{(3)} \hat{g}_{1}+T_{1}^{(3)} \hat{g}_{0}\right] \\
& -\frac{4 i}{4 \pi \lambda}\left[T_{0}^{(4)} \hat{g}_{1}+T_{1}^{(4)} \hat{g}_{0}\right]-\frac{3 i}{4 \pi \lambda} E_{1}+\frac{15}{4 \pi \lambda^{2}} \hat{T}_{1} .
\end{aligned}
$$

In Proposition 3.4, the coefficients $r_{0}$ and $r_{1}$ are deduced from (33) for $m=5$.

Now, introduce a sequence of components of integral operators $\left(\mathcal{A}_{i}^{(n)}\right)_{n \geq 0}$, defined for any $\varphi \in L^{2}(\partial \Omega)^{3}$ by:

$$
\left(\mathcal{A}^{(n)} \varphi\right)_{i}(x)=\int_{\partial \Omega} \Gamma_{i j}^{(n)}(x, y) \varphi_{j}(y) d \sigma(y), \quad \text { for } i, j \in\{1,2,3\} \text { and } n \geq 0 .
$$

Using previous results, il is clear that we know explecitely the first terms $\mathcal{A}_{i}^{(0)}, \mathcal{A}_{i}^{(1)}$. For any positive integer $N$, we can by recursive method get the term $\mathcal{A}_{i}^{(N)}$. Then, the following theorem holds. 
Theorem 3.1 Let $\mathcal{A}^{\delta}$ be the operator defined by (10). Let $\Psi_{\delta}(x)$ be the diffeomorphism defined as in Lemma 2.2. Let $N$ be a positive integer. There exists a positive constant $C$ depending only on $N$, and $\|\rho\|_{C^{2}}$ such that for any $\tilde{\varphi} \in L^{2}\left(\partial \Omega_{\delta}\right)^{3}$ and $i, j \in\{1,2,3\}$, the $i^{\text {th }}$-component $\mathcal{A}_{i}^{\delta}$ defined by (13) satisfies:

$$
\left\|\left(\mathcal{A}^{\delta} \tilde{\varphi}\right)_{i} o \Psi_{\delta}-\left(\mathcal{A}^{(0)} \varphi\right)_{i}-\sum_{n=1}^{N} \delta^{n}\left(\mathcal{A}^{(n)} \varphi\right)_{i}\right\|_{L^{2}(\partial \Omega)} \leq C \delta^{N+1}\|\varphi\|_{L^{2}(\partial \Omega)^{3}}
$$

where $\varphi=\tilde{\varphi} o \Psi_{\delta}$.

\subsection{Asymptotic expansion of the eigenelements}

To develop asymptotic behaviors of eigenvalues and eigenfunctions with respect to the parameter of perturbation $\delta$, we may use the results of Theorem 3.1. Then, the following asymptotic expansion related to the operator $\mathcal{A}_{\delta}$ appears clearly.

Proposition 3.5 Suppose that we have all hypothesis of Theorem 3.1. Then, the operator $\mathcal{A}^{\delta}(\lambda)$ defined by (10) can be expanded uniformly for $x \in \partial \Omega$ as follows:

$$
\mathcal{A}^{\delta}(\lambda) \varphi=\mathcal{A}^{(0)}(\lambda) \varphi+\delta \mathcal{A}^{(1)}(\lambda) \varphi+\delta^{2} \mathcal{A}^{(2)}(\lambda) \varphi+\cdots ; \quad \text { as } \delta \rightarrow 0,
$$

where $\varphi \in L^{2}(\partial \Omega)^{3}$, the $i^{\text {th }}$-component of the first term is given by

$$
\left(\mathcal{A}_{i}^{(0)}(\lambda) \varphi\right)_{i}(x)=\int_{\partial \Omega} \Gamma_{i j}^{(0)}(x, y) \varphi_{j}(y) d \sigma(y), \quad \text { for } i, j \in\{1,2,3\}
$$

and more generally, the $i^{\text {th }}$-component of the term with order $n$ is given by

$$
\left(\mathcal{A}^{(n)}(\lambda) \varphi\right)_{i}(x)=\int_{\partial \Omega} \Gamma_{i j}^{(n)}(x, y) \varphi_{j}(y) d \sigma(y), \quad \text { for } i, j \in\{1,2,3\} \text { and } n \geq 1 .
$$

The coefficients $\Gamma_{i j}^{(n)}$ are given by 49).

Let $a_{j}(\delta)$ denotes:

$$
a_{j}(\delta)=\frac{1}{2 i \pi} \operatorname{tr} \int_{\partial D_{\epsilon_{0}}}\left(\lambda-\lambda_{0}\right)^{j}\left(\mathcal{A}^{\delta}\right)^{-1}(\lambda) \partial_{\lambda} \mathcal{A}^{\delta}(\lambda) \varphi d \lambda,
$$

where $\varphi \in L^{2}(\partial \Omega)^{3}$. The functions $a_{j}(\delta)$ is analytic in a complex neighborhood of 0 and satisfies: $\overline{a_{j}(\delta)}=a_{j}(\bar{\delta})$. The following main result holds.

Proposition 3.6 Suppose that $\lambda_{0}$ is an eigenvalue with multiplicity 1 of the eigenvalue problem (11). Let the operator $\mathcal{A}^{\delta}$ be defined by (10). Then, there exists a small positive number $\delta_{0}$ such that the eigenvalue $\lambda(\delta)$ is analytic in $]-\delta_{0}, \delta_{0}$ [ and satisfy:

$$
\lambda(\delta)=\lambda_{0}+\delta \lambda_{1}+\sum_{n \geq 2} \lambda_{n} \delta^{n}
$$

where the first coefficients are given explicitly by:

$$
\left\{\begin{array}{l}
\lambda_{1}=\frac{1}{2 i \pi} \operatorname{tr} \int_{\partial D_{\epsilon_{0}}}\left(\lambda-\lambda_{0}\right)\left[\left(\mathcal{A}^{(0)}\right)^{-1}(\lambda) \mathcal{A}^{(1)}(\lambda)\left(\mathcal{A}^{(0)}\right)^{-1}(\lambda) \partial_{\lambda} \mathcal{A}^{(0)}(\lambda)\right] \varphi d \lambda, \\
\lambda_{2}=\frac{1}{2 i \pi} \operatorname{tr} \int_{\partial D_{\epsilon_{0}}}\left(\lambda-\lambda_{0}\right)\left[\left(\mathcal{A}^{(0)}\right)^{-1} \mathcal{A}^{(1)}\left(\mathcal{A}^{(0)}\right)^{-1} \partial_{\lambda} \mathcal{A}^{(1)}+\left(\mathcal{A}^{(0)}\right)^{-1} \mathcal{A}^{(2)}\left(\mathcal{A}^{(0)}\right)^{-1} \partial_{\lambda} \mathcal{A}^{(0)}+\right. \\
\left.\left(\mathcal{A}^{(0)}\right)^{-1}\left(\mathcal{A}^{(1)}\left(\mathcal{A}^{(0)}\right)^{-1}\right)^{2} \partial_{\lambda} \mathcal{A}^{(0)}\right] \varphi d \lambda,
\end{array}\right.
$$

where $\varphi \in L^{2}(\partial \Omega)^{3}$ 
Proof. Let $\lambda_{\delta}$ be the eigenvalue of the eigenvalue problem (4) and let $\varphi \in L^{2}(\partial \Omega)^{3}$. Then, if we take the curl of the first equation in (4), we see that there exists a function $w=w\left(v_{\delta}\right)$ called vorticity associated to $v_{\delta}$ such that

$$
\begin{cases}\Delta w+\lambda_{\delta} w=0 & \text { in } \Omega \\ v=0 & \text { in } \partial \Omega\end{cases}
$$

That is, $w$ is an eigenfunction of the negative Laplacian, but with boundary conditions on the velocity $v_{\delta}$.

Next, let $u_{\delta}$ be the stream function for $v_{\delta}$ given as in Lemma 2.10 of [8]. Then $w=\Delta u_{\delta}$ and $\nabla u_{\delta}=0$ on $\partial \Omega$. Since $u_{\delta}$ is determined only up to a constant we can then assume that $u_{\delta}=0$ on $\partial \Omega$.

Thus, $u_{\delta}$ satisfies the following eigenvalue problem for the Dirichlet biharmonic operator:

$$
\begin{cases}\Delta \Delta u_{\delta}+\lambda_{\delta} \Delta u_{\delta}=0 & \text { in } \Omega \\ u_{\delta}=0 & \text { in } \partial \Omega\end{cases}
$$

Note that Temam [16] exploits the similar correspondence between the Stokes problem and the biharmonic problem in the proof of the regularity of solutions to the Stokes system and to justify several results. Moreover, as pointed out by Ashbaugh in [4], there is a similar correspondence between the eigenvalue problems for the Dirichlet Laplacian and system (55) with the boundary condition $\nabla \cdot u_{\delta}=0$ replaced by $\Delta u_{\delta}=0$.

Then, one can exploit this correspondence to use the approach used in [3] to develop an asymptotic expansion for the eigenvalue.

So that, it is well known that there exits a polynomial-valued function $\delta \mapsto \mathcal{Q}_{\delta}(\lambda)$ of degree 1 , analytic in $]-\delta_{0}, \delta_{0}$ [ and of the form:

$$
\mathcal{Q}_{\delta}(\lambda)=\lambda-a_{1}(\delta)
$$

such that the perturbation $\lambda_{\delta}-\lambda_{0}$ is precisely its zero. For the existence of $\mathcal{Q}_{\delta}$ one can follow the general approach used, for example, in [3] for the case of Laplace operator. Writing:

$$
\mathcal{Q}_{\delta}\left(\lambda_{\delta}-\lambda_{0}\right)=0
$$

Then we have

$$
\lambda_{\delta}-\lambda_{0}=a_{1}(\delta)
$$

Therefore, by (52) we have

$$
\lambda_{\delta}-\lambda_{0}=\frac{1}{2 i \pi} \operatorname{tr} \int_{\partial D_{\epsilon_{0}}}\left(\lambda-\lambda_{0}\right)\left(\mathcal{A}^{\delta}\right)^{-1}(\lambda) \partial_{\lambda} \mathcal{A}^{\delta}(\lambda) \varphi d \lambda .
$$

On the other hand, for $\delta$ in a small neighborhood of 0 , the following Neumann series converges uniformly with respect to $\lambda$ in $\partial D_{\epsilon_{0}}$ :

$$
\left(\mathcal{A}^{\delta}\right)^{-1}(\lambda)=\left(\mathcal{A}^{(0)}\right)^{-1}(\lambda)+\sum_{k=1}^{\infty}\left(\mathcal{A}^{(0)}\right)^{-1}(\lambda)\left[\left(\mathcal{A}^{(0)}(\lambda)-\mathcal{A}^{\delta}(\lambda)\right)\left(\mathcal{A}^{(0)}\right)^{-1}(\lambda)\right]^{k} .
$$

So that,

$$
\lambda_{\delta}-\lambda_{0}=\frac{1}{2 i \pi} \operatorname{tr} \int_{\partial D_{\epsilon_{0}}}\left(\lambda-\lambda_{0}\right)\left(\mathcal{A}^{(0)}\right)^{-1}(\lambda) \partial_{\lambda} \mathcal{A}^{\delta}(\lambda) \varphi d \lambda+
$$




$$
\frac{1}{2 i \pi} \operatorname{tr} \int_{\partial D_{\epsilon_{0}}}\left(\lambda-\lambda_{0}\right) \sum_{k=1}^{\infty}\left(\mathcal{A}^{(0)}\right)^{-1}(\lambda)\left[\left(\mathcal{A}^{(0)}(\lambda)-\mathcal{A}^{\delta}(\lambda)\right)\left(\mathcal{A}^{(0)}\right)^{-1}(\lambda)\right]^{k} \partial_{\lambda} \mathcal{A}^{\delta}(\lambda) \varphi d \lambda
$$

By using (14), we find that $\frac{1}{2 i \pi} \operatorname{tr} \int_{\partial D_{\epsilon_{0}}}\left(\lambda-\lambda_{0}\right)\left(\mathcal{A}^{(0)}\right)^{-1}(\lambda) \partial_{\lambda} \mathcal{A}^{\delta}(\lambda) \varphi d \lambda=0$. This result is a direct consequence of the fact that $\mathcal{R}_{0}(\lambda)$ and $\partial_{\lambda} \mathcal{A}^{\delta}(\lambda)$ are holomorphic in the variable $\lambda$.

Now we have:

$$
\begin{gathered}
\lambda_{\delta}-\lambda_{0}=\frac{1}{2 i \pi} \operatorname{tr} \int_{\partial D_{\epsilon_{0}}}\left(\lambda-\lambda_{0}\right)\left(\mathcal{A}^{(0)}\right)^{-1}(\lambda)\left[\left(\mathcal{A}^{(0)}(\lambda)-\mathcal{A}^{\delta}(\lambda)\right)\left(\mathcal{A}^{(0)}\right)^{-1}(\lambda)\right] \partial_{\lambda} \mathcal{A}^{\delta}(\lambda) \varphi d \lambda+ \\
\frac{1}{2 i \pi} \operatorname{tr} \int_{\partial D_{\epsilon_{0}}}\left(\lambda-\lambda_{0}\right)\left(\mathcal{A}^{(0)}\right)^{-1}(\lambda)\left[\left(\mathcal{A}^{(0)}(\lambda)-\mathcal{A}^{\delta}(\lambda)\right)\left(\mathcal{A}^{(0)}\right)^{-1}(\lambda)\right]^{2} \partial_{\lambda} \mathcal{A}^{\delta}(\lambda) \varphi d \lambda+ \\
\frac{1}{2 i \pi} \operatorname{tr} \int_{\partial D_{\epsilon_{0}}}\left(\lambda-\lambda_{0}\right) \sum_{k \geq 3}\left(\mathcal{A}^{(0)}\right)^{-1}(\lambda)\left[\left(\mathcal{A}^{(0)}(\lambda)-\mathcal{A}^{\delta}(\lambda)\right)\left(\mathcal{A}^{(0)}\right)^{-1}(\lambda)\right]^{k} \partial_{\lambda} \mathcal{A}^{\delta}(\lambda) \varphi d \lambda .
\end{gathered}
$$

Inserting expression (51) into above relation, we may get:

$$
\begin{aligned}
\lambda_{\delta}-\lambda_{0}= & \frac{1}{2 i \pi} \operatorname{tr} \int_{\partial D_{\epsilon_{0}}}\left(\lambda-\lambda_{0}\right)\left(\mathcal{A}^{(0)}\right)^{-1}\left[\delta \mathcal{A}^{(1)}\left(\mathcal{A}^{(0)}\right)^{-1}+\delta^{2} \mathcal{A}^{(2)}\left(\mathcal{A}^{(0)}\right)^{-1}+\cdots\right]\left(\partial_{\lambda} \mathcal{A}^{(0)}+\delta \partial_{\lambda} \mathcal{A}^{(1)}\right. \\
& \left.+\delta^{2} \partial_{\lambda} \mathcal{A}^{(2)}+\cdots\right) \varphi d \lambda+\frac{1}{2 i \pi} \operatorname{tr} \int_{\partial D_{\epsilon_{0}}}\left(\lambda-\lambda_{0}\right)\left(\mathcal{A}^{(0)}\right)^{-1}\left[\delta \mathcal{A}^{(1)}\left(\mathcal{A}^{(0)}\right)^{-1}+\right. \\
& \left.\delta^{2} \mathcal{A}^{(2)}\left(\mathcal{A}^{(0)}\right)^{-1}+\cdots\right]^{2}\left(\partial_{\lambda} \mathcal{A}^{(0)}+\delta \partial_{\lambda} \mathcal{A}^{(1)}+\delta^{2} \partial_{\lambda} \mathcal{A}^{(2)}+\cdots\right) \varphi d \lambda+\cdots
\end{aligned}
$$

If we collect the same powers of $\delta$, then we get the desired results.

Define,

$$
\mathcal{B}_{\delta}(\lambda) \varphi(x)=(\mathcal{W}(\lambda) \varphi)\left(\Psi_{\delta}^{-1}\right)\left(\Psi_{\delta}(x)\right),
$$

where $\varphi \in L^{2}(\partial \Omega)$ and $\Psi_{\delta}$ given by Section 1, and $\mathcal{W}(\lambda)$ is the operator associated to hydrodynamic double layer potential [10, 11, 17]. Then, the following main results hold.

Theorem 3.2 Let $\mathcal{A}_{\delta}$ be the operator defined by (10), and $\mathcal{M}_{\delta}=\mathcal{A}_{\delta}+\mathcal{B}_{\delta}$. Let $\mathcal{O}_{0}$ be a bounded neighborhood of $\bar{\Omega}$ in $\mathbb{R}^{3}$. Then there exists a constant $\delta_{1}>0$ smaller than $\delta_{0}$ such that the eigenfunction $v(\delta)$ corresponding to the eigenvalue, $\lambda(\delta)$, in $\left(H^{1}\left(\Omega_{\delta}\right)\right)^{3} \cap$ $H\left(\Omega_{\delta}\right)$ can be chosen to depend holomorphically in $\left.(x, \delta) \in \mathcal{O}_{0} \times\right]-\delta_{1}, \delta_{1}[$. Moreover this eigenfunction satisfies the following asymptotic formulae

$$
v(x ; \delta)=v_{0}(x)+\sum_{n \geq 1} v_{n}(x) \delta^{n},
$$

where the function $v_{0}$ is the eigenfunction solution of (1) associated to $\lambda_{0}$. The terms $v_{n}$ are computed from the Taylor coefficients of the operator valued function $\mathcal{M}_{\delta}$ and of those of the function $a(\delta)=\left(a_{i j}(\delta)\right)_{1 \leq i, j \leq 3}$. 
Proof. From [10, 12] we deduce that there exists a continuous function $\varphi(t, \delta)$, which is analytic in $\left.\mathbb{R}^{2} \times\right]-\delta_{0}, \delta_{0}[$ and such that

$$
v_{\delta}(x)=\mathcal{S}\left(\lambda_{\delta}\right) \varphi+\mathcal{W}\left(\lambda_{\delta}\right) \varphi, \quad x \in \Omega
$$

solves the eigenvalue problem (4). Moreover, the function given by

$$
U(\delta)(x)=\mathcal{M}(\lambda(\delta)) \varphi\left(\Psi^{-1}, \delta\right)
$$

satisfies the eigenvalue problem (4) in $\Omega_{\delta}$ with the boundary conditions: $\left.U(\delta)\right|_{\partial \Omega_{\delta}}=0$. Here, $\mathcal{M}(\lambda(\delta)) \varphi\left(\Psi^{-1}, \delta\right)=\mathcal{M}_{\delta}(\lambda) \varphi$.

Now, by (59), we deduce that $v(x ; \delta)=U(\delta)(x)=\mathcal{M}(\lambda(\delta)) \varphi\left(\Psi^{-1}, \delta\right)$ is jointly analytic with respect to $(x, \delta)$ in $\left.\left\{\left\|x-\Psi_{\delta=0}(y)\right\| \leq z_{0}\right\} \times\right]-\delta_{0}, \delta_{0}\left[\right.$, where $z_{0}$ is a positive constant. The function $v(x ; \delta)$ is jointly analytic in the variables $\left.(x, \delta) \in \mathcal{O}_{0} \times\right]-\delta_{0}, \delta_{0}[$.

We shall now give the asymptotic expansion of the function $v(x ; \delta)$ when $\delta$ tends to 0 . Integral equation (7) gives us

$$
v(\delta)(x)=\int_{\partial \Omega} M\left(\lambda(\delta),\left|x-\Psi_{\delta}(y)\right|\right) \varphi(y, \delta)\left|\nabla \Psi_{\delta}(y)\right| d \sigma(y),
$$

where $M$ is the kernel of the operator $\mathcal{M}_{\delta}$. The perturbed eigenvalue $\lambda(\delta)$ is in a small neighborhood of $\lambda_{0}$ for small values of $\delta$. Then we have the following Taylor expansion

$$
M\left(\lambda(\delta),\left|x-\Psi_{\delta}(y)\right|\right)\left|\nabla \Psi_{\delta}(y)\right|=M\left(\lambda_{0},|x-\Psi(y)|\right)|\nabla \Psi(y)|+\sum_{k \geq 1} \delta^{k} M_{k}(x, y),
$$

which holds uniformly in $x \in \overline{\mathcal{O}}_{0}$ and $y \in \partial \Omega$. The analyticity of the function $\varphi(y, \delta)$ with respect to $\delta$ immediately gives

$$
\varphi(t, \delta)=\varphi_{0}(y)+\sum_{k \geq 1} \delta^{k} \varphi_{k}(y)
$$

uniformly in $y \in \partial \Omega$. Substituting the last two asymptotics into (60) we find

$$
v(x ; \delta)=v(x ; \delta=0)+\sum_{k \geq 1} \delta^{k}\left[\sum_{n=1}^{k} \int_{\partial \Omega} \varphi_{k-n}(y) M_{n}(x, y) d \sigma(y)\right] .
$$

The next result provide us with the asymptotic expansion of the eigenpressures.

Corollary 3.1 Suppose that we have all hypothesis of Theorem (3.2). Then the eigenpressures $p_{\delta}$ solution of (4) have the following uniform asymptotic expansion:

$$
p_{\delta}(x)=p_{0}(x)+\sum_{n \geq 1} p_{n}(x) \delta^{n},
$$

where the function $p_{0}$ is the eigenpressure solution of (1) associated to $\lambda_{0}$. The terms $p_{n}$ are computed from the Taylor coefficients $\lambda_{n}$ and $v_{n}=\left(v_{n}^{1}, v_{n}^{2}, v_{n}^{3}\right)$ as follows:

$$
p_{n}\left(. ; x_{i} ; .\right)=\int \Delta v_{n}^{i} d x_{i}+\sum_{k=0}^{n} \lambda_{k} \int v_{n-k}^{i} d x_{i}, \quad \text { where } i=1,2,3 .
$$


Proof. From system (44) we have

$$
\nabla \cdot p_{\delta}=\Delta v_{\delta}+\lambda_{\delta} v_{\delta}
$$

. Hence, we can expand the function $p_{\delta}$ in powers of $\delta$ as we have done for $\lambda_{\delta}$ and $v_{\delta}$. Moreover, we have:

$$
\partial_{i} p_{\delta}=\Delta v_{\delta}^{i}+\lambda_{\delta} v_{\delta}^{i}, \quad \text { for } i=1,2,3 .
$$

To get the coefficients of the formula (64) one can insert both asymptotic expansions (53) and (58) into relation (65), and integrate with the convenable variable.

\section{References}

[1] J.H. Albert, Genericity of simple eigenvalues for elliptic pde's, Proc. Amer. Math. Soc., 48 (1975), 413-418.

[2] J.H. Albert, Topology of the nodal and critical points sets for eigenfunctions of elliptic operators, Ph. D. Thesis, M. I. T., Boston MA, 1971.

[3] H. Ammari, F. Triki, Splitting of resonant and scattering frequencies under shape deformation. J. Differ. Equations 202, No. 2, (2004) 231-255.

[4] M. S. Ashbaugh, On universal inequalities for the low eigenvalues of the buckling problem. In Partial differential equations and inverse problems, volume 362 of Contemp. Math., pages 13-31. Amer. Math. Soc., Providence, RI, 2004.

[5] I. Babuska, J.E. Osborn, Eigenvalue Problems, in: P.G. Ciarlet, J.L. Lions (Eds.), Handbook of Numerical Analysis, vol. II, Finite Element Method (Part I), NorthHolland, Amsterdam, 1991, pp. 641?787.

[6] N. Depauw, Solutions des équations de Navier Stokes incompressibles dans un domaine exterieur Rev. Mat. Iberoam. 17(2001) 21-68.

[7] T. Kato, Perturbation Theory for Linear Operators, Springer-Verlag, Berlin, Heidelberg, 1980.

[8] James. P. Kelliher, Eigenvalues of the Stokes operator versus the Dirichlet Laplacian in the plane. Pac. J. Math. 244, No. 1, (2010) 99-132.

[9] A. Khelifi, Asymptotic property and convergence estimation for the eigenelements of the Laplace operator. Appl. Anal. 86, No. 10, (2007) 1249-1264.

[10] M. Kohr, The interior Neumann problem for the Stokes resolvent system in a bounded domain in $\mathbb{R}^{n}$, Arch. Mech., 59, 3, (2007), 283-304.

[11] O. A. Ladyzhenskaya, Mathematical theory of the viscous incompressible id. Fizmathiz, Moscow, 1961, p. 203.

[12] D. Medkova and W. Varnhorn, Boundary value problems for the Stokes equations with jumps in open sets, Applicable Analysis, 87: 7, (2008, )829- 849.

[13] J. H. Ortega, and E. Zuazua, Generic simplicity of the eigenvalues of the stokes system in two soace dimensions, Advances in Differential Equations. Volume 6, Number 8, (2001), 987-1023.

[14] Jia. Shanghu, Xie. Hehu, Yin. Xiaobo and Gao. Shaoqin Approximation and eigenvalue extrapolation of Stokes eigenvalue problem by nonconforming finite element methods. Applications of Mathematics, vol. 54 (2009), issue 1, pp. 1-15 
[15] R. Temam, Navier-Stokes Equations, Theory and Numerical Analysis, Elsevier Science Publishers B. V., Amsterdam, 1984.

[16] R. Temam, Navier-Stokes equations. AMS Chelsea Publishing, Providence, RI, 2001. Theory and numerical analysis, Reprint of the 1984 edition.

[17] W. Varnhorn, The Stokes equations, Akademie Verlag, Berlin 1994. 